\title{
Design and Experimental Research on Natural Ventilation Windows Based on Active and Passive Noise Reduction Technologies
}

\author{
Dan LIU1, a, Ziyong CHEN ${ }^{1, b}$, Yunkang $\mathrm{JI}^{1, \mathrm{c}}$, Hejun $\mathrm{LI}^{1, \mathrm{~d}}$, Xibing TUO ${ }^{1, \mathrm{e}}$ and \\ Weigang ZHENG ${ }^{2, f^{*}}$ \\ ${ }^{1}$ School of Energy and Power Engineering Institute, Wuhan University of Technology, Wuhan \\ Hubei 430063, China \\ ${ }^{2}$ Engineering Training Center, Wuhan university of technology, Wuhan Hubei 430063, China \\ a785448528@qq.com, b992139655@qq.com, '839846027@qq.com, d437319792@qq.com, \\ e1979079277@qq.com, ‘ miaozhen13520@163.com
}

Keywords: Natural ventilation window; Active and passive noise control; Experimental research Abstract. To solve the problem of that the existing sound insulation window cannot control the low-medium frequency noise effectively, active and passive noise control technologies are used in the internal and external staggered open channel to broaden the noise reduction frequency range of the existing sound insulation window and ensure natural ventilation. The experiment research shows that active noise control technology can bring more than 15dBA extra noise reduction at low-medium frequency and passive noise reduction technology can bring more than 10dBA extra noise reduction at medium-high frequency, the whole window ensured natural ventilation at the same time achieves $42 \mathrm{dBA}$ noise reduction .

\section{Introduction}

In recent years, traffic noise in urban has a great impact on indoor environment and people's physical and mental health, in this reason, reducing traffic noise spreading into indoor and improving the indoor environment has been paid close attention.According to China Indoor Environmental Monitoring Committee, $90 \%$ of the indoor noise is coming through the window, so improving the sound insulation performance of the window is one of the most direct and effective methods to solve the problem of indoor noise.However, the existing sound insulation window cannot be well isolated from low-medium frequency noise, so it cannot solve the urban traffic noise effectively.

On the basis of the existing sound insulation window, a natural ventilation window based on active and passive noise reduction technology is designed.This improvement can broaden the noise reduction frequency range of the existing sound insulation window and optimize the noise reduction performance, which are verified through experimentation.

\section{Structure of the Natural Ventilation Window based on Active and Passive Noise Reduction Technology.}

To assure indoor sunlight, this window is composed of the upper part of the noise control passage and the lower part of the ordinary casement window, and broken bridge aluminum alloy and tempered insulation glass are used as the materials, which is shown in Fig. 1.The noise control passage is composed of outer hung window, inner hung window and bottom air outlet with fence type.Active and passive noise reduction device is installed in the upper noise control passage, which is shown in Fig. 2. 


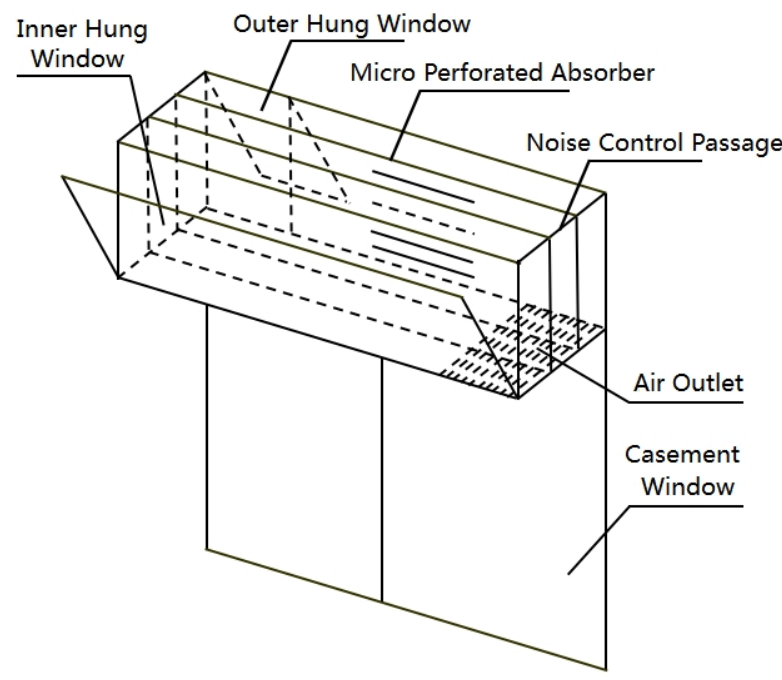

Fig. 1 Structure of the window

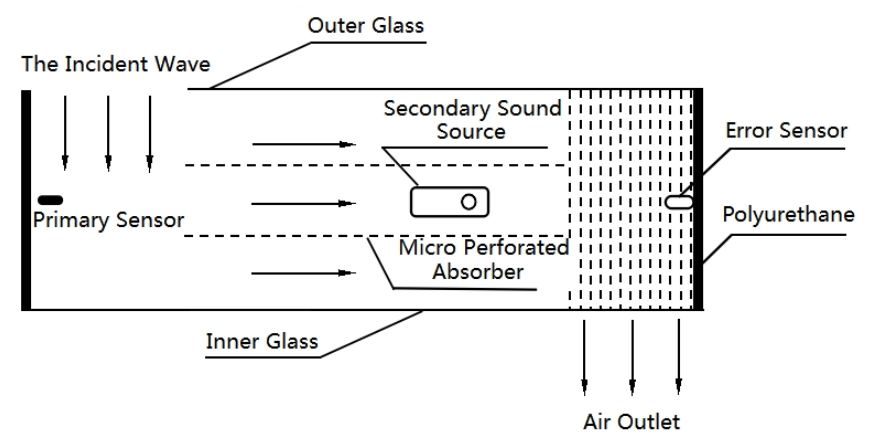

Fig. 2 Structure of noise control passage

Furthermore, the cross-sectional area size of the air outlet is determined by indoor per-person ventilation.According to the standard of indoor air quality [1], people will not feel uncomfortable when the indoor per-person ventilation is more than $30 \mathrm{~m}^{3} / \mathrm{h}$. There is a window which air outlet cross-sectional area is $0.011 \mathrm{~m}^{2}$, and indoor per-person ventilation can obtain $28 \mathrm{~m}^{3} / \mathrm{h}$ which is meeting the requirement basically when outdoor wind speed is $1.5 \sim 2.5 \mathrm{~m} / \mathrm{s}$ [2]. On this basis, the air outlet size of this window is designed to $0.0225 \mathrm{~m}^{2}$ which well meets the requirement of indoor per-person ventilation under the condition that the annual average wind speed is $2.8 \mathrm{~m} / \mathrm{s}$ in Wuhan.

\section{Principle of Active and Passive Noise Reduction Technology}

Indoor noise can get effective control by using the active and passive noise reduction technology.Active noise control device adopts the single channel adaptive active noise control system, passive noise control device adopts the sound absorption materials with porous structure and micro perforation plate structure.

Single channel adaptive active noise control system is shown in Fig. 3.Primary sound field is formed in the noise control passage when outdoor noise enters it.Primary sensor collects noise signal and transmits it to controller which can generate a signal of secondary sound sources by adaptive algorithm.Power amplifier amplifies this signal and drives the secondary sound source making noise and producing secondary sound field in the noise control passage.Error sensor receives the sound pressure of the primary and secondary sound field at the same time, the error signal is formed after the combination of those two signals. Adaptive algorithm adjusts the weights of the controller according to the preset targets and changes the strength of the secondary signal, including amplitude, frequency and phase after error signal is transmitted to controller.Then, primary waves and secondary waves cause destructive interference, which is shown in Fig. 4.This process continues to proceed and the system will tend to stable eventually, then achieve the purpose of eliminating low-medium frequency noise [3].

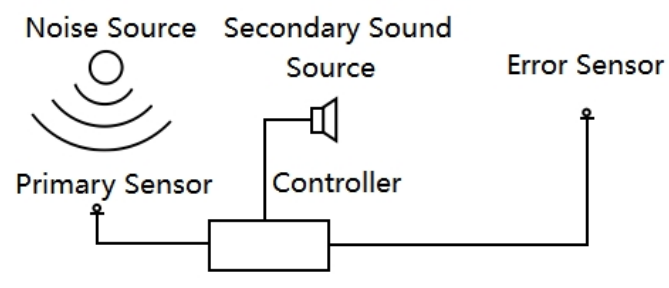

Fig. 3 Adaptive active control system

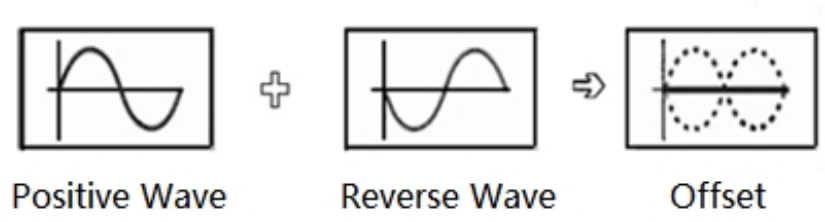

Fig. 4 Principle of acoustic wave offset 
Passive noise reduction device consists of two parts, one part is the porous sound absorbing polyurethane arranged around the noise control passage, which can cause viscous and friction effect with acoustic wave, resulting most of the sound energy converted into heat and thus the acoustic energy is attenuated [4], the other part is the PETG micro perforated panel arranged transverse, which can cause resonance with air, resulting the air around the perforated plate produced vibration and friction and formed the absorption peak, thus achieving sound absorption [5].In this way, the sound absorption performance of this two kinds of materials are combined and the medium-high frequency noise can be effectively controlled.

\section{Experiment of the noise reduction performance}

The experiment is conducted in Institute of Acoustics, Nanjing University.The sound insulation performance of the active noise control device and passive noise control device is verified through this experimentation, the window and measurement system are shown in Fig. 5.

The window is installed on the wall in the middle of the acoustic source room and receiving room, the outer hung window of the noise control passage opens towards acoustic source room, the inner hung window and air outlet open towards receiving room. When doing experiment, the outer hung window is opened and the inner hung window is closed.The noise source, generated white noise in the range of $0 \sim 1600 \mathrm{HZ}$ and used to simulate the urban traffic noise, is put in acoustic source room. The sensor, located at outside the window $10 \mathrm{~cm}$ and used to measure sound pressure in acoustic source room, is put at test point 1 .The other two sensors, respectively located at outside the window $10 \mathrm{~cm}$ and $2 \mathrm{~m}$ and used to measure sound pressure in receiving room, are respectively put at test point 2 and test point 3 .

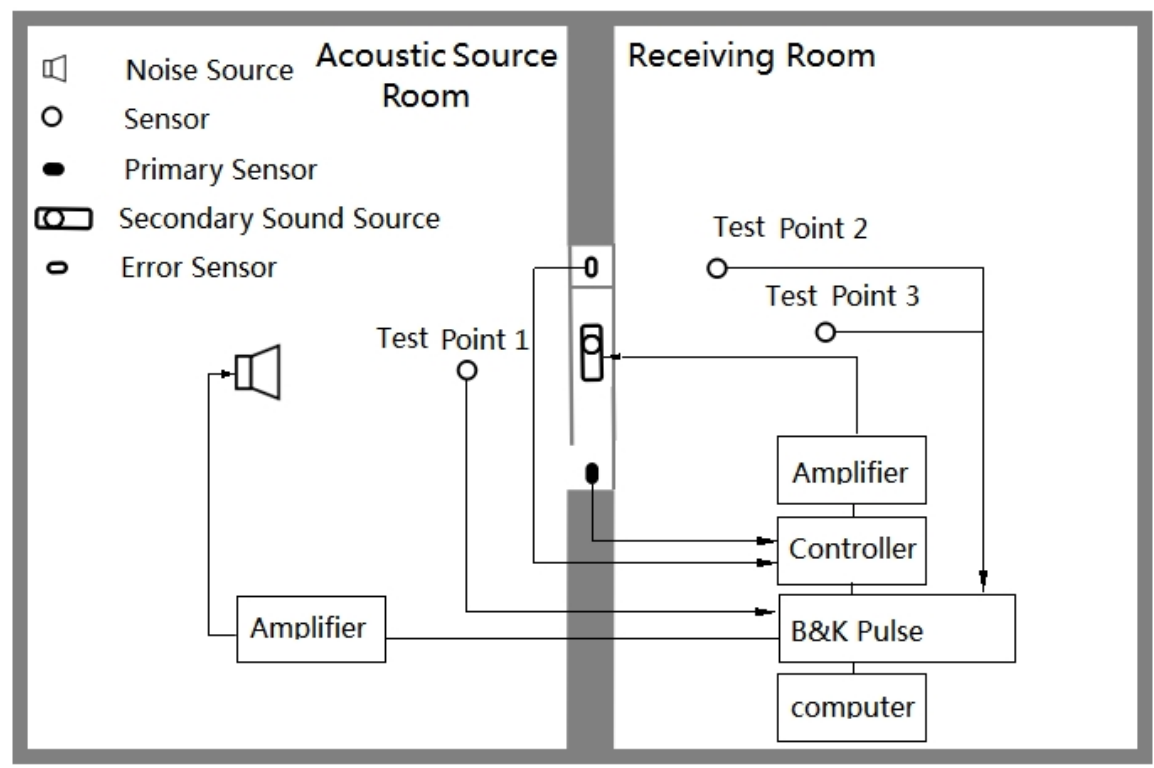

Fig. 5 The actively and passively controlled window and measurement system used in the experiments

Measure and analyze the noise reduction performance of the active noise control device. A condition that active noise control system works alone is designed in this experiment, which is designed to verify that if it has great effect on low-medium frequency noise.

When doing experiment, passive noise reduction device is removed, casement window and inner hung window are closed, and outer hung window is opened to the most.At this time, because noise control passage is directly connected with outside, most of the sound energy is through the air inlet into noise control passage and then from air outlet to receiving room, so primary sensor is installed at the position of air inlet and error sensor is installed at the position of air outlet.Considered the position of the secondary sound source has a great influence on the non-linear distortion of secondary sound 
source, the causality of the feed-forward adaptive control system, and the real-time and stabilization of the whole system, secondary sound source is put at the position between primary sensor and error sensor, where slightly close to air outlet [6].This position is shown in Fig.2.

The noise spectrum of the test point 2 is shown in Fig. 6, red line shows the real-time sound pressure when active noise control system is closed, green line shows the real-time sound pressure when active noise control system is opened.According to this spectrum, the low-medium frequency noise in the range of 100 450HZ can be effectively controlled by active noise control system, and there is $15 \mathrm{dBA}$ extra noise reduction at this range of frequency.This experiment verifies that active noise control system has great effect on low-medium frequency noise.

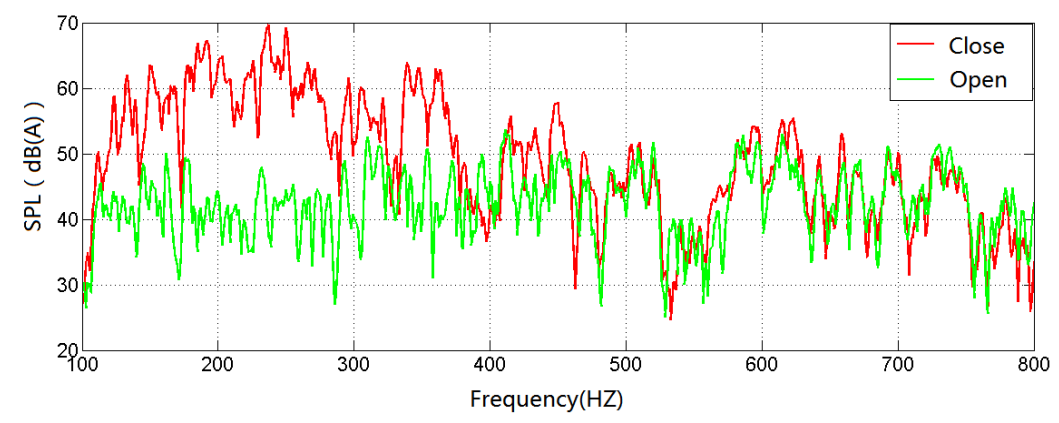

Fig. 6 Active noise reduction spectrum

Measure and Analyze the Noise Reduction Performance of the Passive Noise Control Device. Related studies have shown that the porous sound absorbing polyurethane has great effect on medium-high noise where the average absorption coefficient is more than 0.5.As the consequences of MAA's theory [7], microperforated panel absorber has the advantages of wide absorption band and high absorption rate.Considering the structure and the size of this window, parameters of micro perforated panel are set to:perforation rate $\mathrm{e}=1 \%$, aperture $\mathrm{d}=0.8 \mathrm{~mm}$, thickness $\mathrm{t}=0.8 \mathrm{~mm}$, back cavity thickness $\mathrm{d}=60 \mathrm{~mm}$.

This experiment is designed to verify the noise reduction performance when this two kinds of sound absorption materials is combined.During the experiment, active noise control device is removed, the outer hung window is opened and the casement window is closed.The noise spectrum is shown in Fig. 7 , which is respectively measured at test point 2 before and after the installation of this two kinds of sound absorption materials.

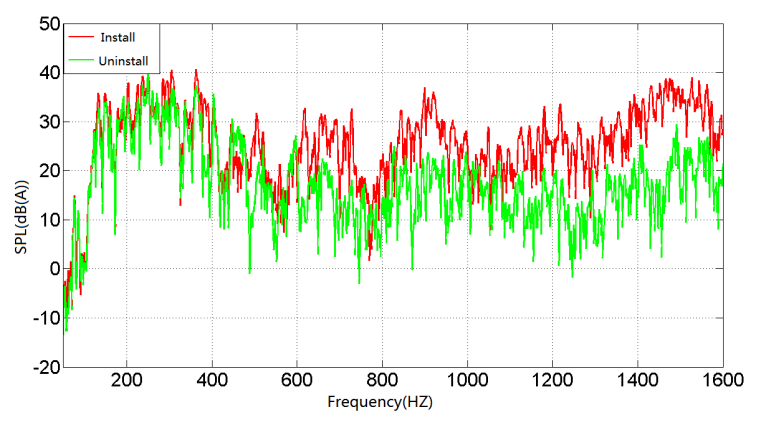

Fig.7 Passive noise reduction spectrum with passive materials

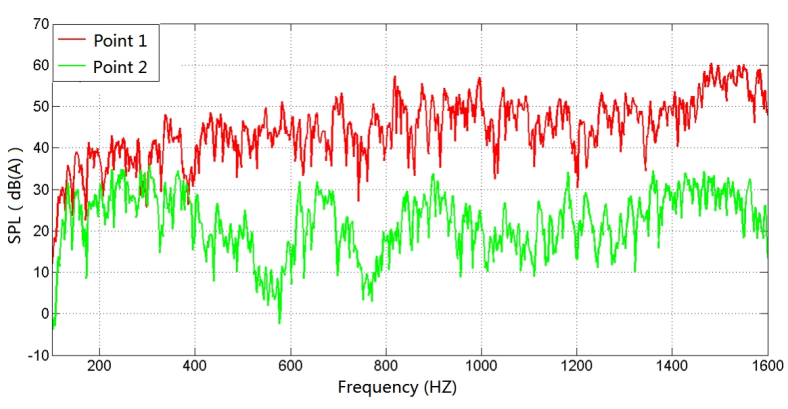

Fig. 8 Spectrum of the noise control passage

Red line shows the real-time sound pressure when sound absorption materials is removed, green line shows the real-time sound pressure when sound absorption materials is installed.According to this spectrum, sound absorption materials has great effect on medium-high frequency noise, and the average noise reduction is more than 10dBA.

In order to further verify the performance of noise reduction when the noise control passage combined with this two kinds of sound absorption materials, noise spectrums measured at test point 1 
and test point 2 are compared, and we find that it has great effect on the noise in the range of 500 1600HZ from Fig. 8, the average noise reduction can up to 25dBA.

Measure and Analyze the Noise Reduction Performance of the Whole Window. According to the results of the experiments, active noise control system has great effect on the low-medium frequency noise in the range of 100 450HZ and the noise control passage combined with the sound absorption materials has great effect on medium-high frequency noise in the range of 500 1600HZ.In order to verify the noise reduction performance of the whole window, active and passive noise control devices are installed, outer hung window is opened, inner hung window and casement window are closed, open the controller and obtain the noise spectrum of the test point 1 and the test point 3 , which is shown in Fig. 9.
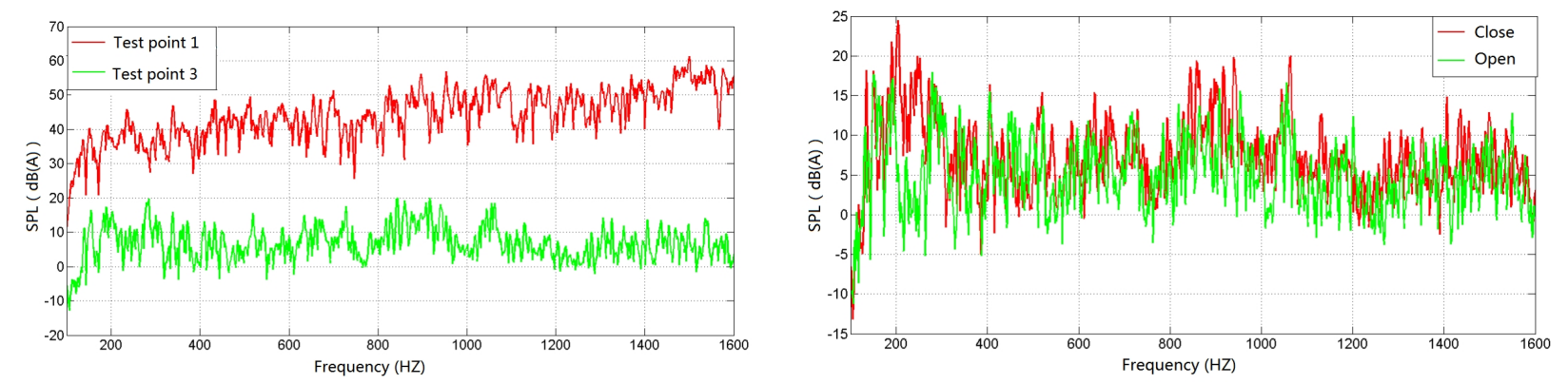

Fig. 9 The noise reduction spectrum of whole window Fig. 10 The noise reduction spectrum at testing point 2 when the window opened and close

Red line shows the real-time sound pressure at the test point 1, green line shows the real-time sound pressure at the test point 3.The analysis of the spectrum shows that the average sound pressure in acoustic source room is $80.5 \mathrm{dBA}$, the average sound pressure in receiving room is $38.3 \mathrm{dBA}$, and the noise reduction of whole window is $42.2 \mathrm{dBA}$.

To further verify the noise reduction performance of the whole window, we also compare the noise spectrum which is respectively measured at test point 2 , in the cases, when the whole window is closed, the outer window and controller is opened.The noise spectrum is shown in Fig. 10, red line shows the real-time sound pressure when the whole window is closed, green line shows the real-time sound pressure when the outer window and controller is opened, and both of the noise is almost equal.This experiment verifies that this window based on active and passive noise reduction technologies not only realizes noise control, but also realizes the natural ventilation, and the noise reduction performance is equal to the case when the whole window is closed.

\section{Conclusions}

In this research, the natural ventilation window, on the basis of the existing sound insulation window, applies active and passive noise reduction technologies, achieves natural ventilation, broadens the noise reduction frequency range, offsets the deficiency of he existing sound insulation window, and improve the noise reduction performance of the whole window.

\section{Acknowledgements}

The project sponsored by national innovation entrepreneurship training program of college students in Wuhan University of Technology.(NO.20151049705004).

\section{References}

[1] GB/T 18883-2002. Indoor air quality standard [S]. In Chinese. 
[2] Wuzhou Yu, Zuomin Wang. The design and experiment on a new type natural ventilation window with high sound insulation [J]. Environmental Engineering, 2008, 26(3):96-99.In Chinese.

[3] Haoxin $\mathrm{Yu}$, KeanChen, Han $\mathrm{Hu}$, Xiyue Ma.Experimental investigation on influence factors of active noise attenuation of sound-insulation ventilation window $[\mathrm{J}]$. Noise and Vibration Control, 2012, 32(3):191-197. In Chinese.

[4] Xiaoqiang Xu, Ruping Yang, Chunxiao Xiong.Research of sound-absorbing polyurethane foam[J]. Aerospace Materials \& Technology, 2011, (2):17-20. In Chinese.

[5] Kean Chen. Active noise control [M]. Beijing: National Defence Industry Press, 2003, pp.71-72. In Chinese.

[6] Song Liu, Haishan Zou, Xiaojun Qiu. Experimental research on natural ventilation windows with active noise attenuation [A]. National conference on acoustic academic environment [C]. Noise and Vibration Control, 2012, (S1): 218-219. In Chinese.

[7] MAA Dahyou.General theory and design of microperforated-panel absorbers [J]. Acta Acustic, 1997, (5): 385-393. In Chinese.

The first author:Dan LIU(1994-),female,Wuhan University of Technology,undergraduate student, mainly study the technology of noise control.

Phone: 13098883504

E-mail: $\underline{785448528 @ q q . c o m}$ 